\title{
Chemically tagging the Galactic disk: abundance patterns of old open clusters
}

\author{
G. M. De Silva ${ }^{1}$, K. C. Freeman ${ }^{2}$ and J. Bland-Hawthorn ${ }^{3}$ \\ ${ }^{1}$ European Southern Observatory, Karl-Schwarzschild Str 2. D-85748 Garching, Germany \\ email: gdesilva@eso.org \\ ${ }^{2}$ Research School of Astronomy and Astrophysics, Mount Stromlo Observatory, Australian \\ National University. ACT 2611. Australia \\ email: kcf@mso.anu.edu . au \\ ${ }^{3}$ Institute of Astronomy, University of Sydney, NSW 2006, Australia \\ email: jbh@physics.usyd.edu.au
}

\begin{abstract}
The long term goal of large-scale chemical tagging is to use stellar elemental abundances as a tracer of dispersed substructures of the Galactic disk. The identification of such lost stellar aggregates and exploring their chemical properties will be key in understanding the formation and evolution of the disk. Present day stellar structures such as open clusters and moving groups are the ideal testing grounds for the viability of chemical tagging, as they are believed to be the remnants of the original larger star-forming aggregates. We examine recent high resolution abundance studies of open clusters to explore the various abundance trends and reassess the prospects of large-scale chemical tagging.
\end{abstract}

Keywords. Galaxy: formation - evolution; open clusters and associations: general

\section{Introduction}

The aim of chemical tagging (Freeman \& Bland-Hawthorn 2002) is to re-construct ancient star-forming aggregates of the Galactic disk, assuming such systems existed from an hierarchical aggregation formation scenario. Observationally the fact that stars are born in rich aggregates numbering hundreds to thousands of stars is supported by many studies from optical, infrared, millimeter and radio surveys (e.g. Carpenter 2000; Meyer et al. 2000; Lada \& Lada 2003). The existence of stellar aggregates at earlier epochs is also observed in the form of old open clusters, stellar associations and moving groups. Further, theoretical hydrodynamical simulations indicate that star formation occurs in groups, where the original gas cloud undergoes fragmentation preventing contraction onto a single star (e.g. Jappsen et al. 2005; Tilley \& Pudritz 2004; Larson 1995). Some clusters stay together for billions of years, whereas others become unbound shortly after the initial star-burst, depending on the star formation efficiency. If stars were not born in aggregates, it would be impossible to identify a given star's birth site.

\section{Open clusters}

Open clusters have historically been used for studying stellar evolution as all stars in a given cluster are coeval. Their key attribute is that they provide a direct time line for investigating change. Young and old open clusters are found in the disk, varying in age from several Myr to over 10 Gyr (see compilation by Dias et al. 2002). The older (>1 Gyr) open clusters are less numerous than their younger counterparts and in general are more massive. These old open clusters are excellent probes of early disk evolution. 
They constitute important fossils and are the likely left-overs of the early star-forming aggregates in the disk. Perhaps only the remnant cores are left behind, while the outer stars have dispersed into the disk.

\subsection{Chemical homogeneity}

It is generally assumed that all stars in a cluster, born from the same parent proto-cluster cloud should contain the same abundance patterns. Theoretically, the high levels of supersonic turbulence linked to star formation in giant molecular clouds (McKee \& Tan 2002), suggests that the interstellar medium of the gas clouds is well mixed and supports the case for chemically homogenous clusters. Observational evidence show high levels of chemical homogeneity in open clusters. High accuracy differential abundance studies for large samples of Hyades open cluster F-K dwarfs show little or no intrinsic abundance scatter for a range of elements (Paulson et al. 2003; De Silva et al. 2006). Such internal homogeneity is also observed within old open clusters (e.g. Collinder 261 De Silva et al. 2007; Carretta et al. 2005, ;). Other old open cluster studies also support the case for internal homogeneity (e.g. Jacobson et al. 2007; Bragaglia et al. 2008), albeit for only a few stars and larger measurement uncertainties.

The observations of homogeneity in old open clusters show that chemical information is preserved within the stars and effects of any external sources of pollution (e.g. from stellar winds or interactions with ISM) are negligible. Abundance differences may arise within cluster stars at various stellar evolutionary stages, e.g. due to internal mixing of elements during the dredge-up phases in giants. We do not expect main sequence dwarf members of a cluster to show such effects. Further, any internal mixing will only affect the lighter elements synthesized within the stars, while the heavier element abundances should remain at their initial levels. It is, however, interesting to note Pasquini et al. (2004)'s study of IC 4651, which shows systematic abundance differences between the main sequence turn-off stars and the giants, although this maybe due to errors in deriving the stellar temperature scales.

\subsection{Cluster sample}

Assuming internal homogeneity holds for most open clusters in the disk, we now compare the different cluster abundance patterns. The mean cluster elemental abundances were taken from several high resolution abundance studies in the literature for clusters with ages greater than the Hyades. The list of clusters and their references are given in Table 1. Figure 1 plots the cluster mean abundance relative to Fe for the various elements studied.

Before the different clusters can be compared we must note that systematic differences are likely to exist due to differences in methodologies and scales. Since the studies are based on different clusters with no overlapping samples, such systematics are difficult to quantify. Where a study included an analysis of a reference star, such as the Sun, we can use the quoted differences as a guide to the expected systematic effects. In other studies, the reference solar abundance levels were simply adopted from past literature sources. This systematic difference arising due to the solar reference values is typically within 0.05 dex.

Systematic uncertainties differ from element to element, depending on how the individual lines were analyzed. We have adopted the published results based on standard LTE analysis to ensure a better comparison, and non-LTE analyses were not included. The employed atomic line data varies between studies and is also gives rise to systematics. In 
Table 1. Open cluster sample

\begin{tabular}{|c|c|c|}
\hline Cluster Name & {$[\mathrm{Fe} / \mathrm{H}]$} & Reference \\
\hline NGC 6253 & 0.46 & Carretta et al. (2007) \\
\hline NGC 6791 & 0.47 & Carretta et al. (2007) \\
\hline NGC 7142 & 0.08 & Jacobson et al. (2007) \\
\hline NGC 6939 & 0.00 & Jacobson et al. (2007) \\
\hline IC 4756 & -0.15 & Jacobson et al. (2007) \\
\hline M 11 & 0.10 & Gonzalez \& Wallerstein (2000) \\
\hline NGC 2324 & -0.17 & Bragaglia et al. (2008) \\
\hline NGC 2477 & 0.07 & Bragaglia et al. (2008) \\
\hline NGC 2660 & 0.04 & Bragaglia et al. (2008) \\
\hline NGC 3960 & 0.02 & Bragaglia et al. (2008) \\
\hline Be 32 & -0.29 & Bragaglia et al. (2008) \\
\hline NGC 6819 & 0.09 & Bragaglia et al. (2001) \\
\hline NGC 7789 & -0.04 & Tautvaišienè et al. (2005) \\
\hline M 67 & -0.03 & Tautvaišiene et al. (2000) \\
\hline NGC 2141 & -0.26 & Yong et al. (2005) \\
\hline Be 31 & -0.40 & Yong et al. (2005) \\
\hline Be 29 & -0.18 & Yong et al. (2005) \\
\hline Be 20 & -0.61 & Yong et al. (2005) \\
\hline Tom 2 & -0.45 & Brown et al. (1996) \\
\hline Mel 71 & -0.30 & Brown et al. (1996) \\
\hline NGC 2243 & -0.48 & Gratton \& Contarini (1994) \\
\hline Mel 66 & -0.38 & Gratton \& Contarini (1994) \\
\hline Cr 261 & -0.03 & De Silva et al. (2007) \\
\hline Hyades & 0.13 & De Silva et al. (2006) \\
\hline
\end{tabular}

differential analyses relative to the Sun or a reference star, the $g f$ values were recalculated for each element and line. Other studies use laboratory measured $g f$ values from various literature sources. Other differences in analyses, such as the use of different model atmospheres and whether the abundance measurements are based on EWs or spectral synthesis also produce systematic variations. Since most of these systematics cannot be accurately quantified, we have not taken them into account and plot the published mean abundance values in Figure 1. The error bars representing the typical measurement errors for the various elements in each study are shown. We refer the reader to the original studies for the individual measurement errors per element per cluster.

Other points to note include the number and type of stars in the different studies. The clusters studied by Bragaglia et al. (2008); Carretta et al. (2007) are based largely on 5-6 red clump stars per cluster, the Yong et al. (2005) and Jacobson et al. (2007) studies on 2-5 red giants per cluster, while other studies are based on main sequence or turn-off dwarfs. As mentioned in section 2.1, we do not expect any effects of stellar evolution for the heavier elements, while the abundances of lighter elements, such as $\mathrm{Na}, \mathrm{Al}, \mathrm{Mg}$ may be affected in the giants by internal mixing. In this case they will no longer represent the cluster's initial abundance levels, which will introduce additional scatter when comparing to dwarf members of other clusters.

\subsection{Abundance signatures}

Figure 1 shows that different clusters have different elemental abundance patterns. There is also significant scatter for many of the elements. As discussed above, some of this scatter is likely to due systematic uncertainties, although there may be intrinsic variations, especially in elements showing excessive scatter. 


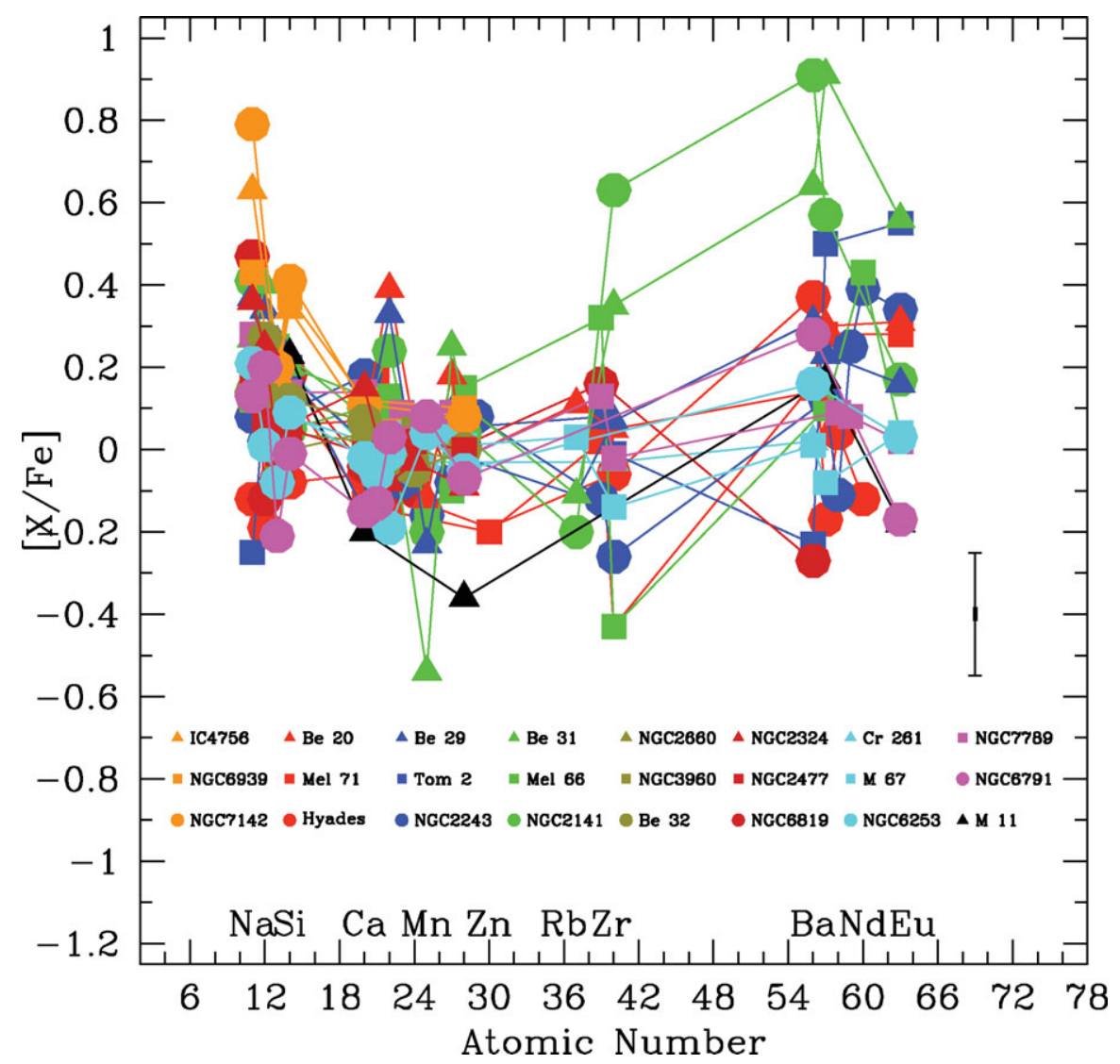

Figure 1. Elemental abundances of old open clusters. Each symbol represents the mean abundance value for individual clusters. The error bars show the typical measurement error. Original references of the cluster data are given in Table 1.

Of the lighter elements, $\mathrm{Na}$ has the highest scatter, where most clusters are $\mathrm{Na}$ enhanced except for two clusters showing sub-solar levels. The other odd Z and alpha elements show cluster to cluster scatter within 0.15 dex with the average abundance being slightly super-solar. Enhanced alpha elements relative to $\mathrm{Fe}$ are indicative of a high rate of star formation where Type II SN dominate over the Type Ia SN. Therefore clusters showing such enhancement is likely to have undergone a phase of rapid star formation in comparison to those which show solar or sub-solar level alpha abundances. Of further interest are the clusters that do not show equal alpha element abundance levels, e.g. with enhanced $\mathrm{Si}$ but deficient in $\mathrm{Ca}$. This may represent some form of localized inhomogeneity unique to the time and site of the clusters' formation. Such abundance signatures will play a major role in large scale chemical tagging, when associating field stars to common origins.

The Fe-peak elements, thought to be produced via Type Ia SN, in general show the least scatter with the average abundance close to solar. This is expected given the abundances are plotted relative to Fe and we expect the Fe-peak elements to follow the Fe abundance. Nevertheless it is interesting to note that $\mathrm{Ti}$ has a larger scatter. $\mathrm{Ti}$ is considered the heaviest of the alpha elements, although by atomic number it falls into the Fe-peak group. It is not considered a pure alpha element either since Type Ia SNe 
also contribute to its production in addition to the dominant Type II SNe. Among the other Fe-peak elements Mn also shows a higher scatter, however this is dominated by a single cluster which is extremely Mn deficient. Note again that Mn could be synthesized in Type II SN as well as from Type Ia, where the yields are metallicity dependent (McWilliam et al. 2003; Shetrone et al. 2003). From these examples it is clear that many of the elements behave differently within their groups. They do not necessarily vary in lock-steps and it is likely that various nucleosynthesis processes are at play.

The heavier s- and r- process elements show the largest scatter of all studied elements. Note the number of data points for these neutron capture elements are much less than for the lighter elements, a sign that they are difficult to measure. The mean abundance levels are super-solar for these elements. The exceptions are $\mathrm{Zr}$ with clusters showing both super and sub-solar $\mathrm{Zr}$ abundance levels, as well as $\mathrm{Rb}$ and Ce, which have sub-solar abundances in the clusters although with only few data points. The s-process elements are synthesized in low neutron flux environments, as in AGB stars and mixed into the ISM by stellar winds. The light s-process elements such as $\mathrm{Zr}$ seem to show a lower abundance compared to the heavier s-process elements such as Ba. Note however the opposite trend is observed for two clusters. Varying trends between $\mathrm{Ba}$, La and $\mathrm{Ce}$ are also seen. The mostly r-process elements such as $\mathrm{Nd}$ and $\mathrm{Eu}$, produced in high flux environments during Type II SNe also show various trends among the open cluster abundances. Further the ratio of s- to r- process element abundance varies from cluster to cluster. We can expect this as both the s- and r-processes contribute at different levels to the production of these neutron capture elements. Similar to the alpha and Fe-peak groups, this further demonstrates that the common group elements do not always vary in lock step. Their various abundance levels highlight the different conditions during the formation of the individual clusters.

\section{Conclusion}

We have used high resolution elemental abundances of old open clusters from the literature to compare the cluster to cluster abundance trends for a large range of elements. We find that different clusters show different abundance levels for a given element, with some elements showing large scatter. Despite systematic uncertainties among the studies which could be the source of much of the abundance scatter, those elements showing a $\sigma>$ 0.2 dex is likely to be an indication of real cluster to cluster abundance variations. Further various element to element abundance patterns were seen among the sample, highlighting the decoupled nature of the elements and the existence of chemical signatures unique to the clusters based on their time and site of formation. An homogenous high resolution abundance study for a range of elements of the Galactic open cluster population (e.g. the BOCCE project, Bragaglia 2007) will provide much valuable insight to further explore unique chemical signatures. This preliminary look, however, suggests that establishing cluster signatures for large scale chemical tagging of the disk is indeed a viable technique.

\section{References}

Bragaglia, A. 2007, ArXiv e-prints, 711, 2171

Bragaglia, A., Sestito, P., Villanova, S., Carretta, E., Randich, S., \& Tosi, M. 2008, A \& A, 480, 79

Bragaglia, A., Carretta, E., Gratton, R. G., Tosi, M., Bonanno, G., Bruno, P., Calì, A., Claudi, R., Cosentino, R., Desidera, S., Farisato, G., Rebeschini, M., \& Scuderi, S. 2001, AJ, 121, 327 
Brown, J. A., Wallerstein, G., Geisler, D., \& Oke, J. B. 1996, AJ, 112, 1551

Carpenter, J. M. 2000, AJ, 120, 3139

Carretta, E., Bragaglia, A., \& Gratton, R. G. 2007, A \& A, 473, 129

Carretta, E., Bragaglia, A., Gratton, R. G., \& Tosi, M. 2005, A\&A, 441, 131

De Silva, G. M., Freeman, K. C., Asplund, M., Bland-Hawthorn, J., Bessell, M. S., \& Collet, R. 2007, AJ, 133, 1161

De Silva, G. M., Freeman, K. C., Bland-Hawthorn, J., Asplund, M., \& Bessell, M. S. 2007, AJ, 133,694

De Silva, G. M., Sneden, C., Paulson, D. B., Asplund, M., Bland-Hawthorn, J., Bessell, M. S., \& Freeman, K. C. 2006, AJ, 131, 455

Dias, W. S., Alessi, B. S., Moitinho, A., \& Lépine, J. R. D. 2002, A\& A, 389, 871

Freeman, K. \& Bland-Hawthorn, J. 2002, ARAA, 40, 487

Gonzalez, G. \& Wallerstein, G. 2000, PASP, 112, 1081

Gratton, R. G. \& Contarini, G. 1994, A\&A, 283, 911

Jacobson, H. R., Friel, E. D., \& Pilachowski, C. A. 2007, AJ, 134, 1216

Jappsen, A.-K., Klessen, R. S., Larson, R. B., Li, Y., \& Mac Low, M.-M. 2005, A\&A, 435, 611

Lada, C. J. \& Lada, E. A., 2003, ARAA, 41, 57

Larson, R. B. 1995, MNRAS, 272, 213

McKee, C. F. \& Tan, J. C. 2002, Nature, 416, 59

McWilliam, A., Rich, R. M., \& Smecker-Hane, T. A. 2003, ApJl, 592, L21

Meyer, M. R., Adams, F. C., Hillenbrand, L. A., Carpenter, J. M., \& Larson, R. B. 2000, Protostars and Planets, 121

Paulson, D. B., Sneden, C., \& Cochran, W. D. 2003, AJ, 125, 3185

Pasquini, L., Randich, S., Zoccali, M., Hill, V., Charbonnel, C., \& Nordström, B. 2004, A \& A, 424,951

Shetrone, M., Venn, K. A., Tolstoy, E., Primas, F., Hill, V., \& Kaufer, A. 2003, AJ, 125, 684

Tautvaišienè, G., Edvardsson, B., Puzeras, E., \& Ilyin, I. 2005, A\&A, 431, 933

Tautvaišiene, G., Edvardsson, B., Tuominen, I., \& Ilyin, I. 2000, A\&A, 360, 499

Tilley, D. A. \& Pudritz, R. E. 2004, MNRAS, 353, 769

Yong, D., Carney, B. W., \& de Almeida, M. L. T. 2005, AJ, 130, 597 